\title{
SAHA down-regulates the expression of indoleamine 2,3-dioxygenase via inhibition of the JAK/STAT1 signaling pathway in gallbladder carcinoma cells
}

\author{
PENG ZHANG $^{1 *}$, GUANMIN JIANG $^{2 *}$, JIAO GAO $^{3}$, LINGLING LI $^{2}$, JUN DU $^{2}$ and XINGYUAN JIAO ${ }^{1}$ \\ ${ }^{1}$ Organ Transplant Center, The First Affiliated Hospital, Sun Yat-sen University, Guangzhou 510080; \\ ${ }^{2}$ Department of Microbial and Biochemical Pharmacy, School of Pharmaceutical Science, Sun Yat-sen University, \\ University Town, Guangzhou 510006; ${ }^{3}$ Department of Hepatobiliary Surgery, The Second Affiliated Hospital, \\ Guangzhou Medical University, Guangzhou 510260, P.R. China
}

Received September 6, 2012; Accepted October 4, 2012

DOI: $10.3892 / o r .2012 .2073$

\begin{abstract}
The aim of the present study was to investigate the role of the JAK/STAT1 signaling pathway in suberoylanilide hydroxamic acid (SAHA)-mediated down-regulation of indoleamine 2,3-dioxygenase (IDO) in gallbladder carcinoma cells. We treated SGC-996 gallbladder carcinoma cells with IFN- $\gamma$ and SAHA. Western blotting was used to detect the expression of IDO, signal transducer and activator of transcription 1 (STAT1) phosphorylation and interferon regulatory factor genes-1 (IRF-1). Confocal microscopy analysis was used to detect STAT1 translocation. Transient transfection and reporter gene assay was used for detecting the activation of $\gamma$-activated sites (GAS) and interferon-stimulated response elements (ISRE). The results revealed that IDO was expressed in SGC-996 cells in a dose- and time-dependent manner when stimulated with IFN- $\gamma$ and SAHA down-regulated the expression of IDO induced by IFN- $\gamma$ in a dose-dependent manner.
\end{abstract}

Correspondence to: Dr Xingyuan Jiao, Organ Transplant Center, The First Affiliated Hospital, Sun Yat-sen University, No. 58 Zhongshan Road 2, Guangzhou 510080, Guangdong Province, P.R. China

E-mail: jiaoxingyuan@hotmail.com

Dr Jun Du, Department of Microbial and Biochemical Pharmacy, School of Pharmaceutical Science, Sun Yat-sen University, No. 132 Waihuandong Road, University Town, Guangzhou 510006, Guangdong Province, P.R. China

E-mail: dujun@mail.sysu.edu.cn

*Contributed equally

Abbreviations: PGC, primary gallbladder carcinoma; HDACI, histone deacetylases inhibitors; SAHA, suberoylanilide hydroxamic acid; IDO, indoleamine 2,3-dioxygenase; IFN- $\gamma$, interferon- $\gamma$; JAK, Janus kinase; STAT1, signal transducer and activator of transcription 1; GAS, $\gamma$-activated sites; ISRE, interferon-stimulated response elements; IRF-1, interferon regulatory factor genes-1

Key words: gallbladder carcinoma, indoleamine 2,3-dioxygenase, SAHA, tumor immune tolerance, JAK/STAT1, IFN- $\gamma$
SAHA blocked the expression of IRF-1 induced by IFN- $\gamma$ and SAHA inhibited IFN- $\gamma$-induced STAT1 phosphorylation and nuclear translocation. In addition, SAHA down-regulated IFN- $\gamma$-induced activation of GAS and ISRE. In conclusion, SAHA down-regulated IDO expression via inhibition of the activation of members of the JAK/STAT1 signaling pathway. Therefore, regulation of the JAK/STAT1 signaling pathway may provide a new gallbladder carcinoma immunotherapeutic strategy to break tumor immune tolerance.

\section{Introduction}

Primary gallbladder carcinoma (PGC) is the most common and aggressive malignancy in the biliary system. The evolution of PGC is a complex multi-step process, including the malignant transformation of normal cells and malignant tumor cell proliferation, invasion and metastasis. This process is not only regulated by oncogenes and anti-oncogenes, but also closely depends on the tumor stromal microenvironment. Due to lacking characteristic signs or symptoms, patients diagnosed as PGC have mostly been in advanced stage with a low resection ratio (1-3). With the in-depth understanding of the immune system and its regulation, immunotherapy has become the focus of tumor treatment due to its promising advantages. Immunotherapy can clean away tumor cells in stationary phase, cancer stem cells and a small amount of residual tumor cells to prevent tumor metastasis and recurrence. However, the clinical outcomes of most immunotherapeutic strategies have been less effective than anticipated. It has been demonstrated that the reasons behind the attenuated clinical outcomes of cancer vaccines were mainly because of the tumor immune tolerance induced by many immune tolerance factors, which originate from the tumor and tumor microenvironment. Indoleamine 2,3-dioxygenase (IDO), as one of the main factors, plays a crucial role in tumor-induced immune tolerance via regulating T-cell function by macrophages and a subset of dendritic cells (4).

IDO is a heme-containing monomeric oxidoreductase that catalyzes the first and rate-limiting step in the degradation of the essential amino acid tryptophan to $\mathrm{N}$-formylkynurenine, and it induces tryptophan starvation and accumulation of 
downstream breakdown products. Because tryptophan is the essential amino acid in the process of T-cell activation and proliferation, tryptophan starvation resulting from IDO consumption inhibits T-cell activation and proliferation. Therefore, $\mathrm{T}$ cells cannot synthesize sufficient protein to proliferate and result in immune tolerance of the local microenvironment $(5,6)$.

IDO has been investigated in pulmonary, hepatocellular, renal, endometrial and nasopharyngeal carcinomas (7-10). The inhibition of IDO expression could significantly suppress tumor growth and promote the anti-tumor activities of various immunotherapeutic agents in relevant animal models $(11,12)$. More importantly, previous studies of our group have reported that IFN- $\gamma$ induces the expression of IDO via the Janus kinase/ signal transducer and activator of transcription 1 (JAK/STAT1) signaling pathway $(13,14)$. Based on these backgrounds, we wondered whether inhibition of the JAK/STAT1 signaling pathway could down-regulate the expression of IDO in gallbladder carcinoma cells and restore the tumor antigen-specific T-cell proliferation, and eventually break tumor immune tolerance and enhance the effect of tumor immunotherapy.

It is widely accepted that the JAK/STAT1 signaling pathway of IDO expression induced by IFN- $\gamma$ is as follows: the interaction of IFN- $\gamma$ with its cell surface receptor leads to the activation of JAK1 and JAK2 (phosphorylation), which in turn phosphorylate and activate STAT1. Phosphorylated STAT1 forms a homodimer and translocates to the nucleus where it binds to and activates IFN- $\gamma$-responsive specific promoters of IDO, including members of the $\gamma$-activated sites (GAS) and the interferon stimulated response elements (ISRE) family of enhancers, and interferon regulatory factor genes-1 (IRF-1) to promote gene transcription $(8,15-17)$. In the current study, we reported that suberoylanilide hydroxamic acid (SAHA) down-regulated the expression of IDO induced by IFN- $\gamma$ in human gallbladder carcinoma (SGC-996) cells and therefore, may provide potential therapeutic strategies in tumor immunotherapy.

SAHA, a kind of histone deacetylases inhibitors (HDACI), is a promising new anticancer agent due to its low toxicity in cancer treatment and protective action against intracellular events including IFN- $\gamma$-mediated signaling transduction $(18,19)$. HDACI are a group of compound regulating the expression of different regulatory genes which are responsible for cell growth, proliferation, apoptosis and regulation of other mechanisms involved in tumor development and growth (20-22).

However, the mechanism of the anti-tumor activity of SAHA remains to be elucidated in more detail. Here, we treated SGC-996 cells with SAHA and IFN- $\gamma$ to investigate the role of inhibition of the JAK/STAT1 signaling pathway by SAHA on the down-regulation of the expression of IDO, in anticipation of providing a new strategy to break tumor immune tolerance in gallbladder carcinoma immunotherapy.

\section{Materials and methods}

Chemicals and reagents. IFN- $\gamma$ and SAHA were respectively purchased from Sigma-Aldrich (Deisenhofen, Germany) and Cayman Chemical (Ann Arbor, MI, USA). Dual-luciferase assay kit and vector (pRL-TK) were purchased from Promega (Madison, WI, USA). Vectors (pGL3-Enhancer-GAS7 and pGL3-Enhancer-ISRE4) were kindly provided by Professor
Jun Du (Sun Yat-sen University, China). Monoclonal antiSTAT1 (9H2) and anti-phospho-Stat1 (Tyr701) antibodies were acquired from a commercial source (Cell Signaling Technology, Beverly, MA). The secondary anti-mouse antibody conjugated to FITC, DAPI dye and Lipofectamine 2000 reagent were purchased from Invitrogen (Carlsbad, CA, USA). The polyclonal rabbit anti-human IDO and monoclonal mouse anti-human IRF-1 antibody (H-8) were products of Santa Cruz Biotechnology Inc. (Santa Cruz, CA, USA).

Cell culture. The human primary gallbladder carcinoma cell line SGC-996 was purchased from the Academy of Life Science, Tongji University (23). Cells were maintained in RPMI-1640 supplemented with $10 \%$ heat-inactivated endotoxin-free fetal bovine serum, $100 \mathrm{~g} / \mathrm{ml}$ streptomycin and $100 \mathrm{U} / \mathrm{ml}$ penicillin under a humidified $5 \% \mathrm{CO}_{2}$ atmosphere at $37^{\circ} \mathrm{C}$ in a $\mathrm{CO}_{2}$ incubator.

Western blot analysis. Western blotting was performed as previously described (13). Briefly, cells were lysed in lysis buffer containing 1\% Nonidet P-40, $20 \mathrm{mM}$ Tris- $\mathrm{HCl}$ (pH 7.6), $0.15 \mathrm{M}$ $\mathrm{NaCl}, 3 \mathrm{mM}$ EDTA, 3 mM EGTA, $1 \mathrm{mM}$ phenylmethylsulfonyl fluoride, $20 \mathrm{mg} / \mathrm{ml}$ aprotinin, and $5 \mathrm{mg} / \mathrm{ml}$ leupeptin. The lysates were cleared by centrifugation and denatured by boiling in Laemmli buffer; equal amounts of protein samples were separated on $10 \%$ sodium dodecylsulfate (SDS)polyacrylamide gels and electrophoretically transferred to nitrocellulose membranes. Following blocking with 5\% non-fat milk at room temperature for $2 \mathrm{~h}$, membranes were incubated overnight with the primary antibody at $1: 1,000$ dilution at $4^{\circ} \mathrm{C}$ and then incubated with a horseradish peroxidase-conjugated secondary antibody at 1:5,000 dilution for $1 \mathrm{~h}$ at room temperature. Specific immune complexes were detected with the Western Blotting Plus Chemiluminescence Reagent (Life Science, Inc., Boston, MA).

Transient transfections and reporter gene assays. For measuring the activation of SAHA on STAT1-dependent transcriptional activation of GAS and ISRE, cells were plated in a 96-well plate at a density of $2.0 \times 10^{4}$ in $500 \mu \mathrm{l}$ of growth medium per well without antibiotics. Twenty-four hours later, cells were $80 \%$ confluent at the time of transfection, and were transfected with $0.2 \mu \mathrm{g}$ DNA/ $/ \mathrm{cm}^{2}$ per plasmid [including objective plasmid and thymidine kinase (TK)] and Lipofectamine 2000 reagent according to the manufacturer's instructions. Transfection efficiency was normalized by co-transfection with pRL-TK. Transcriptional activity was determined by a luminometer, using a Dual-Luciferase Assay kit. Results are displayed as the ratio between the activity of the reporter plasmid and pRL-TK.

Confocal microscopy for STAT1. Cells were grown on $18 \times 18 \mathrm{~cm}$ coverslips in a 6-well plate, serum starved for $16 \mathrm{~h}$, then stimulated with or without $10 \mu \mathrm{M}$ SAHA for $2 \mathrm{~h}$. Followed by being treated with or without $500 \mathrm{U} / \mathrm{ml} \mathrm{IFN}-\gamma$ for $30 \mathrm{~min}$, cells were fixed in $4 \%$ paraformaldehyde and permeabilized with $1 \%$ Triton X-100 (prepared from 30\% stock solution) for $30 \mathrm{~min}$ equally, then blocked with goat serum for $30 \mathrm{~min}$ at $37^{\circ} \mathrm{C}$ and incubated with anti-STAT1 antibody at 1:100 dilution for $1 \mathrm{~h}$ at $37^{\circ} \mathrm{C}$. Coverslips were washed with PBS and incubated 
A

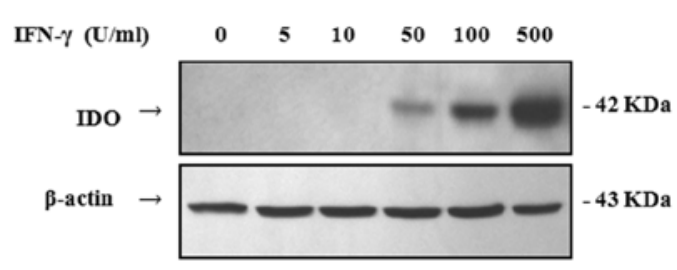

C

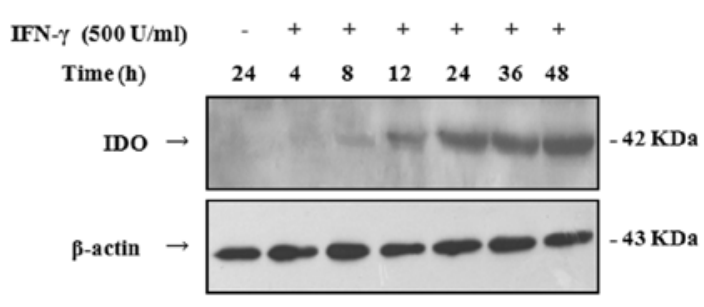

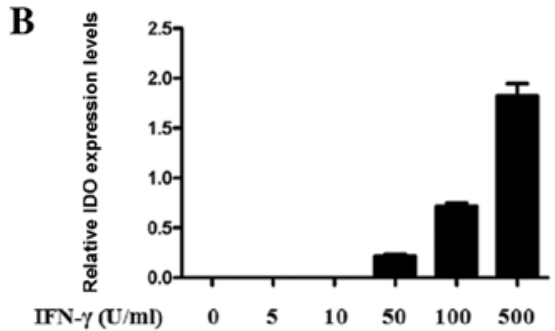

D

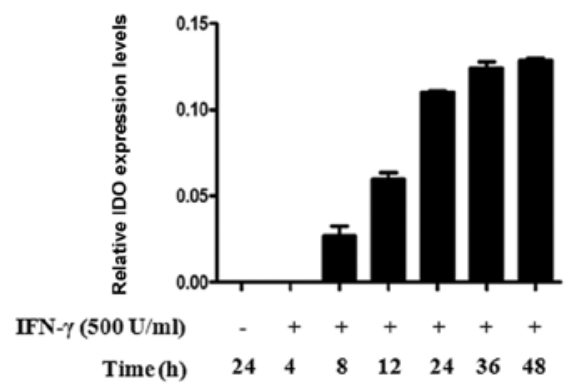

Figure 1. IFN- $\gamma$ induces the expression of IDO in a dose- and time-dependent manner. SGC-996 cells were treated with reagents as indicated and IDO expression was detected by Western blotting. $\beta$-actin served as the loading control. (A and B) Cells were treated with different concentrations of IFN- $\gamma$ for 24 h. (C and D) Cells were treated with $500 \mathrm{U} / \mathrm{ml} \mathrm{IFN}-\gamma$ for the indicated times.

with a secondary anti-mouse antibody conjugated to FITC at 1:1000 dilution for $1 \mathrm{~h}$ at $37^{\circ} \mathrm{C}$. After being washed 3 times with PBS, the cells were incubated with DAPI $(10 \mu \mathrm{g} / \mathrm{ml})$ for $10 \mathrm{~min}$ to visualize cell nuclei. The coverslips were mounted to microscopic slides and covered with glycerol jelly, and then the slides were analyzed by a Confocol Laser Scanning Microscope (LSM 710, Carl Zeiss, Germany) to analyze nuclear translocation of STAT1.

Statistical analysis. All values are expressed as the means \pm SEM of two independent experiments unless otherwise specified. We analyzed data by two-tailed unpaired Student's t-tests between the two groups and by one-way ANOVA followed by the Bonferroni test for multiple comparisons. These analyses were performed using the GraphPad Prism Software Version 5.0 (GraphPad Software Inc., La Jolla, CA). $\mathrm{P}<0.05$ was considered statistically significant.

\section{Results}

IDO expression induced by IFN- $\gamma$ is dose-and time-dependent in SGC-996 cells. To investigate the effect of IFN- $\gamma$ on the expression of IDO in SGC-996 cells, we performed Western blot analysis to explore the effects of varying concentrations and incubation periods of IFN- $\gamma$ on the expression of IDO. SGC-996 cells were treated with IFN- $\gamma$ at different doses for $24 \mathrm{~h}$ or $500 \mathrm{U} / \mathrm{ml} \mathrm{IFN-} \gamma$ for different times. There was no IDO expression observed without IFN- $\gamma$ treatment, but IFN- $\gamma$ significantly enhanced IDO expression in a dose-dependent manner, beginning with $50 \mathrm{U} / \mathrm{ml}$ (Fig. $1 \mathrm{~A}$ and $\mathrm{B}$ ). In addition, IDO was induced by exposure to $500 \mathrm{U} / \mathrm{ml}$ IFN- $\gamma$ for $8 \mathrm{~h}$ and was enhanced by continuous exposure to IFN- $\gamma$ (Fig. 1C and D). Thus, IDO expression in SGC-996 cells was an inducible event, and the expression of IDO induced by IFN- $\gamma$ was doseand time-dependent.
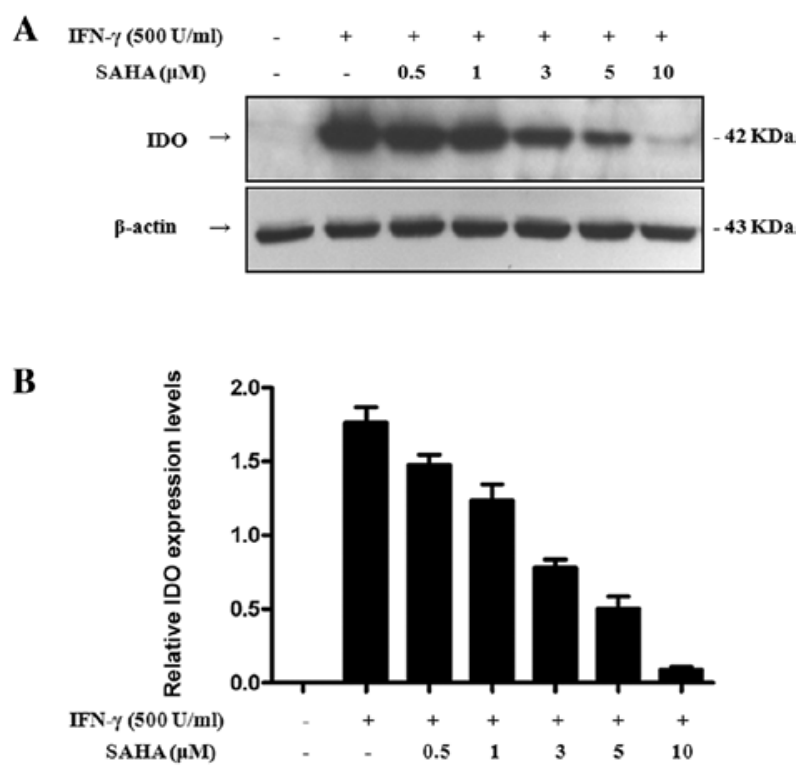

Figure 2. SAHA down-regulates the IFN- $\gamma$-induced expression of IDO in a dose-dependent manner. SGC-996 cells were treated with reagents as indicated. Western blot analysis of IDO expression compared to the reference bands of $\beta$-actin. Cells were pre-treated with various concentrations of SAHA for $2 \mathrm{~h}$ and then treated with $500 \mathrm{U} / \mathrm{ml} \mathrm{IFN}-\gamma$ for $24 \mathrm{~h}$.

SAHA down-regulates the expression of IDO induced by $I F N-\gamma$ in a dose-dependent manner. We further explored the influence of SAHA on IDO expression. SGC-996 cells were pre-treated with various concentrations of SAHA for $2 \mathrm{~h}$, and then treated with IFN- $\gamma$ for $24 \mathrm{~h}$. We performed Western blot analysis to detect IDO expression in total cell lysates. Treatment with SAHA (up to $0.5 \mu \mathrm{M}$ ) dramatically reduced IDO expression induced by $500 \mathrm{U} / \mathrm{ml} \mathrm{IFN}-\gamma$ (Fig. 2). With increasing concentrations of SAHA, IDO expression decreased. IDO expression was almost completely inhibited at a concentration 
A

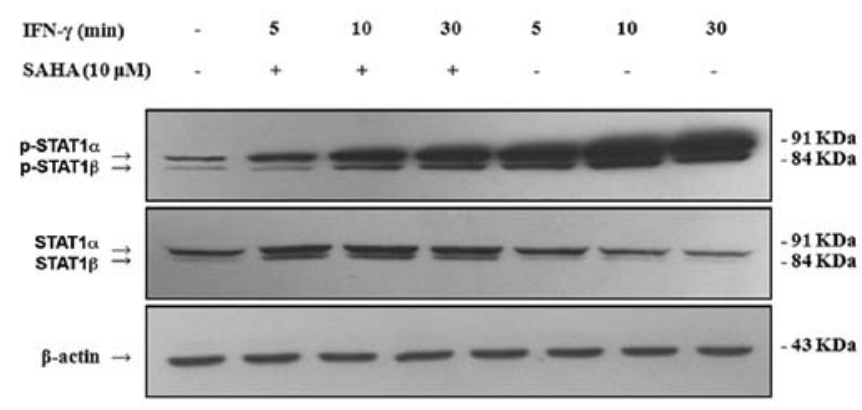

B
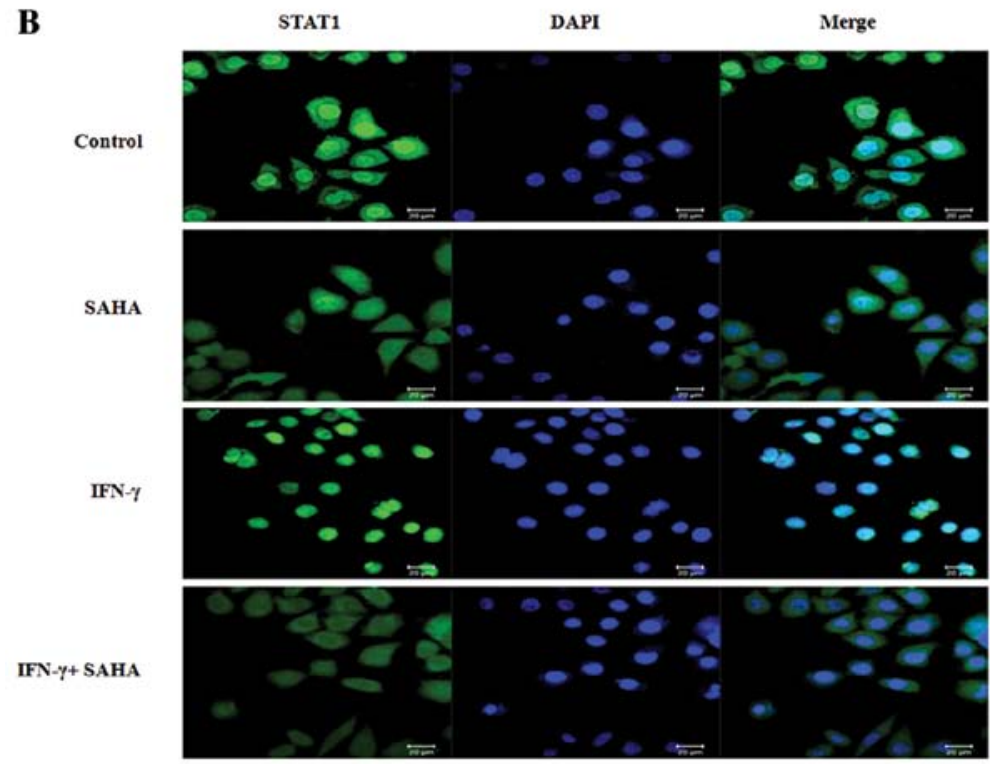

Figure 3. SAHA inhibits STAT1 phosphorylation and nuclear translocation. (A) Cells were treated with or without $10 \mu \mathrm{M}$ SAHA for $2 \mathrm{~h}$ and then treated with $500 \mathrm{U} / \mathrm{ml}$ IFN- $\gamma$ for 5, 10 or $30 \mathrm{~min}$. The phosphorylation of STAT1 and total STAT1 were detected by Western blotting with an anti-pSTAT1-Tyr701 antibody and an anti-STAT1 antibody, respectively. $\beta$-actin served as the loading control. Similar results were obtained in three independent experiments. (B) Cells were grown on chamber slides and were pre-treated with or without SAHA (10 $\mu \mathrm{M})$ for $2 \mathrm{~h}$, followed by treatment with or without IFN- $\gamma(500 \mathrm{U} / \mathrm{ml})$ for $30 \mathrm{~min}$. Immunofluorescence and confocal microscopy were performed as described in Materials and methods. Scale bar, $20 \mu \mathrm{m}$.

of $10 \mu \mathrm{M}$. Therefore, SAHA inhibited the expression of IDO induced by IFN- $\gamma$ in a dose-dependent manner.

SAHA inhibits IFN- $\gamma$-induced STAT1 phosphorylation and nuclear translocation. IFN- $\gamma$ is known to induce IDO expression via activation of STAT1 (24). STAT1 has two isoforms, STAT1 $\alpha(91 \mathrm{kDa})$ and STAT1 $\beta(84 \mathrm{kDa})$. Both isoforms can be activated by IFN- $\gamma$. Phosphorylation of STAT1 at Tyr701 induces STAT1 dimerization, nuclear translocation and DNA binding. STAT1 phosphorylation acts as an active transcription factor in the nucleus. Thus, we wondered whether SAHA blocked STAT1 Tyr701 phosphorylation and inhibited its nuclear translocation. SGC-996 cells were pre-incubated with or without $10 \mu \mathrm{M}$ SAHA for $2 \mathrm{~h}$ and then treated with $500 \mathrm{U} / \mathrm{ml} \mathrm{IFN}-\gamma$ for 5,10 or $30 \mathrm{~min}$. We performed Western blot analysis to detect the phosphorylation of STAT1 with an anti-phospho-STAT1 (Tyr701) antibody as described above. Phosphorylated STAT1 was expressed without stimulation by IFN- $\gamma$ in SGC-996 cells. Stimulation of cells with IFN- $\gamma$ for 5 min alone brought about a rapid increase in tyrosine phosphorylation of STAT1. The expression of phosphorylated STAT1 increased with the stimulation time of IFN- $\gamma$. However, SAHA significantly inhibited this increase (Fig. 3A). To further investigate whether SAHA inhibited STAT1 nuclear transloca- tion, SGC-996 cells were grown on coverslips, pre-incubated with or without $10 \mu \mathrm{M}$ SAHA for $2 \mathrm{~h}$, and then treated with or without $500 \mathrm{U} / \mathrm{ml} \mathrm{IFN-} \gamma$ for $30 \mathrm{~min}$. STAT1 nuclear localization was assessed by confocal microscopy with an anti-STAT1 antibody. STAT1 was localized exclusively in the cytoplasm of untreated cells, and SAHA did not alter the basal subcellular localization of STAT1. On the contrary, cells treated with IFN- $\gamma$ for $30 \mathrm{~min}$ showed a significant nuclear translocation of STAT1. However, IFN- $\gamma$-induced nuclear translocation of STAT1 was considerably reduced after pre-treatment with SAHA for $2 \mathrm{~h}$ (Fig. 3B). In summary, our data suggested that SAHA inhibits phosphorylation and nuclear translocation of STAT1, and this inhibition may result in down-regulation of IDO expression.

SAHA blocks IFN- $\gamma$-induced activation of GAS and ISRE. Following the determination that SAHA inhibits the tyrosine phosphorylation of STAT1 and its translocation to nucleus, we examined whether the inhibitory effect of SAHA could block the transcriptional activation of STAT1 in the nucleus. The reporter gene plasmids pGL3-Enhancer-GAS7-luc and pGL3Enhancer-ISRE4-luc were transfected into SGC-996 cells. The transfection efficiency was normalized by co-transfection with pRL-TK. The results showed that IFN- $\gamma$ significantly 
A

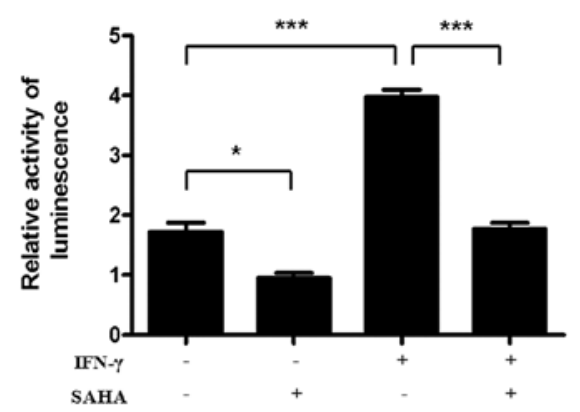

$\mathbf{B}$

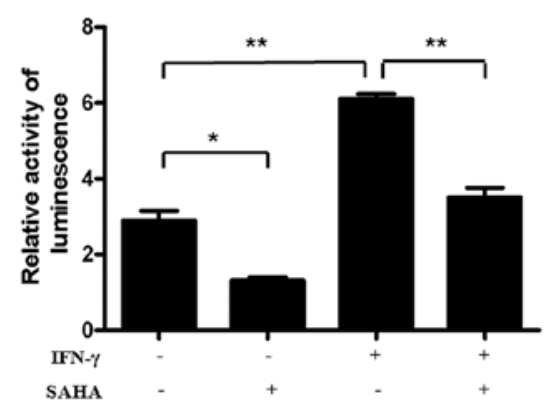

Figure 4. Effect of SAHA on IFN- $\gamma$-induced activation of GAS and ISRE. Cells were transfected with pGL3-Enhancer-GAS7-luc (A), pGL3-Enhancer-ISRE4luc (B) reporter plasmids and treated with $10 \mu \mathrm{M}$ SAHA for $2 \mathrm{~h}$, followed by treatment with $500 \mathrm{U} / \mathrm{ml}$ IFN- $\gamma$ for $24 \mathrm{~h}$. Luminescence was measured with a luminometer. pRL-TK plasmids were used for correcting the transfection efficiency. Results are expressed as the ratio between the activity of the reporter plasmid and pRL-TK. ${ }^{*} \mathrm{P}<0.05 ;^{* *} \mathrm{P}<0.01$ and ${ }^{* * *} \mathrm{P}<0.001$. Similar results were obtained from two independent experiments.

A

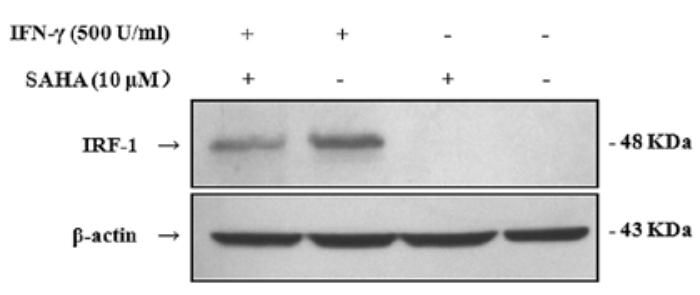

B

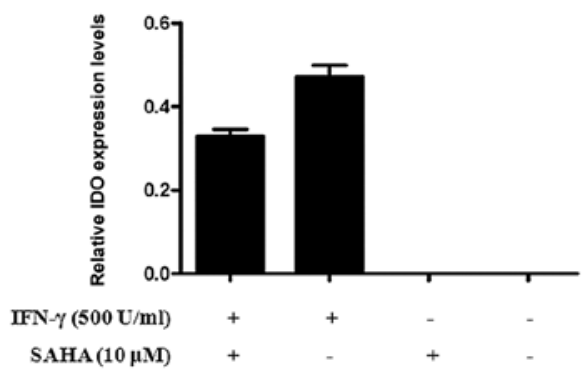

Figure 5. Effect of SAHA on IFN- $\gamma$-induced activation of IRF-1 protein. Cells were pre-treated with or without $10 \mu \mathrm{M} \mathrm{SAHA} \mathrm{for} 2 \mathrm{~h}$ and then treated with or without $500 \mathrm{U} / \mathrm{ml} \mathrm{IFN}-\gamma$ for $8 \mathrm{~h}$. IRF-1 protein in total cell lysates was detected by Western blotting. $\beta$-actin served as the loading control.

enhanced the activity of the pGL3-Enhancer-GAS7-luc and the pGL3-Enhancer-ISRE4-luc via Dual-Glo-Luciferase analysis. Furthermore, SAHA sharply inhibited the activity of pGL3-Enhancer-GAS7-luc and pGL3-Enhancer-ISRE4-luc (Fig. 4). These facts suggested that SAHA down-regulated IFN- $\gamma$-induced expression of IDO via blocking STAT1-driven transcriptional activity, mainly the activity of GAS and ISRE.

SAHA down-regulates the expression of the IRF-1 protein induced by IFN- $\gamma$. IRF-1 is an essential factor for IFN- $\gamma$ induced expression of IDO (8). Therefore, we attempted to determine whether SAHA affected the expression of the IRF-1 protein. Cells were pre-treated with or without $10 \mu \mathrm{M}$ SAHA for $2 \mathrm{~h}$ and then treated with or without $500 \mathrm{U} / \mathrm{ml} \mathrm{IFN}-\gamma$ for $8 \mathrm{~h}$. We used Western blot analysis to detect IRF-1 protein in total cell lysates with a monoclonal mouse anti-human IRF-1 antibody. The results showed that IFN- $\gamma$ dramatically enhanced IRF-1 expression, but SAHA inhibited the expression of IRF-1 which was induced by IFN- $\gamma$ (Fig. 5). It could therefore be concluded that SAHA down-regulated the expression of IDO via blocking activation of IRF-1.

\section{Discussion}

IDO is an important immunogenic regulatory factor in the process of tumor development and tumor immune tolerance $(25,26)$. Overexpression of IDO is closely related to poor prognosis of patients with malignant tumors (27). Furthermore, it is well known that IFN- $\gamma$ induces IDO expression via the JAK/ STAT1 signaling pathway. Consequently, blocking of the JAK/ STAT1 signaling pathway to break immune tolerance may present an option to improve the clinical immunotherapeutic effect in gallbladder carcinoma treatments. However, possible strategies to induce this inhibition remain to be figured out.

IDO has been investigated in many tumors other than gallbladder carcinoma as described above. Therefore, we first detected whether IDO was expressed in SGC-996 cells. In the present study, we demonstrated that IDO was not expressed without IFN $-\gamma$ treatment. However, after stimulation with $50 \mathrm{U} / \mathrm{ml} \mathrm{IFN}-\gamma$ for $24 \mathrm{~h}$ or $500 \mathrm{U} / \mathrm{ml}$ IFN- $\gamma$ for $8 \mathrm{~h}$, the expression of IDO could be significantly detected. IDO expression induced by IFN- $\gamma$ was dose- and time-dependent in the gallbladder carcinoma SGC-996 cells.

Based on the fact that IDO is expressed in SGC-996 cells, we intended to find whether SAHA could inhibit IDO expression in order to improve the immunotherapy of gallbladder carcinoma. In this study, we discovered that SAHA down-regulated the expression of IDO in a dose-dependant manner. Our observations suggest that SAHA may be used in the clinic to improve the efficiency of gallbladder carcinoma immunotherapy.

The IDO gene had a putative promoter region, and this promoter contains a series of putative transcription factor binding sites, including two ISRE regions, a GAS sequence near ISRE-1 and ISRE-2, and two AP-1 binding sites $(28,29)$. In the process of the IFN- $\gamma$-induced expression of IDO, STAT1 
acts as a critical transcriptional factor. STAT1 is phosphorylated at the cytoplasmic tail of the IFN- $\gamma$ receptor, dimerizes and translocates to the nucleus, where it binds GAS to activate IDO and IRF-1 gene expression directly. On the other hand, STAT1 contributes indirectly to the activation of IDO via inducing the production of IRF-1, which binds to ISRE-1 and ISRE-2 elements in the IDO regulatory region resulting in activation of IDO gene expression (8,17,30-32).

According to our data, SAHA inhibited the IFN- $\gamma$-induced phosphorylation of STAT1, which plays an important role in the regulation of IDO gene transcription. The inhibition of STAT1 nuclear translocation resulted in an increase of STAT1 accumulation in the cytoplasm and disruption of the regulation of the IDO gene transcription, directly down-regulating the expression of IDO. In addition, we analyzed the roles of GAS and ISRE, which regulated the expression of IDO in SGC-996 cells. Cells treated with IFN- $\gamma$ significantly induced transcriptional activity of the reporter plasmids pGL3-Enhancer-GAS7 and pGL3-Enhancer-ISRE4 (Fig. 4), suggesting the importance of GAS and ISRE in the IFN- $\gamma$-induced expression of IDO.

Furthermore, SAHA significantly inhibited IFN- $\gamma$ induced transcriptional activation of the reporter plasmid pGL3-Enhancer-GAS7 and pGL3-Enhancer-ISRE4 (Fig. 4). This phenomenon could be explained by the fact that SAHA inhibited STAT1 phosphorylation and nuclear translocation (Fig. 3A), which was important for STAT1 binding of GAS and ISRE to activate the IDO expression. Inhibition of GAS activation could directly down-regulate the expression of IDO. Unlike GAS, activation of ISRE required both phosphorylated STAT1 and IRF-1. Thus, the fact that SAHA inhibited the activation of ISRE could result from the inhibition of phosphorylated STAT1 and IRF-1. The IRF-1 promoter region contains GAS elements which are important for IRF-1 expression. In this study we found that SAHA decreased the IRF-1 protein level induced by IFN- $\gamma$ (Fig. 5). This phenomenon could be explained by the fact that SAHA inhibited STAT1 phosphorylation and activation of GAS, followed by down-regulation of the expression of IRF-1 protein.

In conclusion, we demonstrated for the first time that SAHA down-regulated the expression of IDO induced by IFN- $\gamma$ via interfering with the phosphorylation and nuclear translocation of STAT1 inhibiting its binding to GAS, ISRE and IRF-1 elements in the IDO regulatory region. These discoveries suggest that the JAK/STAT1 signaling pathway plays a key role in the expression of IDO in gallbladder carcinoma cells. Therefore, regulating JAK/STAT1 signaling pathway may provide a new gallbladder carcinoma immunotherapeutic strategy to break tumor immune tolerance and contribute to the development of clinical cancer immunotherapy.

\section{Acknowledgements}

This study was funded by the National Natural Science Foundation of China (no. 30840096, no. 30873032 and no. 81071712), the National Science and Technology Major Project of China (no. 2009ZX09103-040), the National Basic Research Program of China (2011CB935800) and the Bureau of Guangdong Provincial Science and Technology (no. 2008A030201007).

\section{References}

1. Jiao XY, Ren JL, Chen HY, et al: Ala499Val (C>T) and Lys939Gln $(\mathrm{A}>\mathrm{C})$ polymorphisms of the XPC gene: their correlation with the risk of primary gallbladder adenocarcinoma: a case-control study in China. Carcinogenesis 32: 496-501, 2011.

2. Jiao XY, Huang JF, Wu SL, et al: HOGG1 Ser326Cys polymorphism and susceptibility to gallbladder cancer in a Chinese population. Int J Cancer 121: 501-505, 2007.

3. Reid KM, Ramos-De la Medina A and Donohue JH: Diagnosis and surgical management of gallbladder cancer: a review. J Gastrointest Surg 11: 671-681, 2007.

4. Munn DH and Mellor AL: Indoleamine 2,3-dioxygenase and tumor-induced tolerance. J Clin Invest 117: 1147-1154, 2007.

5. Takikawa O: Biochemical and medical aspects of the indoleamine 2,3-dioxygenase-initiated 1-tryptophan metabolism. Biochem Biophys Res Commun 338: 12-19, 2005.

6. Soliman H, Mediavilla-Varela M and Antonia S: Indoleamine 2,3-dioxygenase: is it an immune suppressor? Cancer J 16: 354-359, 2010.

7. Karanikas V, Zamanakou M, Kerenidi T, et al: Indoleamine 2,3-dioxygenase (IDO) expression in lung cancer. Cancer Biol Ther 6: 1258-1262, 2007.

8. Pine R: Convergence of TNFalpha and IFNgamma signalling pathways through synergistic induction of IRF-1/ISGF-2 is mediated by a composite GAS/kappaB promoter element. Nucleic Acids Res 25: 4346-4354, 1997.

9. Yoshida N, Ino K, Ishida Y, et al: Overexpression of indoleamine 2,3-dioxygenase in human endometrial carcinoma cells induces rapid tumor growth in a mouse xenograft model. Clin Cancer Res 14: 7251-7259, 2008

10. Liu P, Xie BL, Cai SH, et al: Expression of indoleamine 2,3-di-oxygenase in nasopharyngeal carcinoma impairs the cytolytic function of peripheral blood lymphocytes. BMC Cancer 9: 416, 2009.

11. Miyazaki T, Moritake K, Yamada K, et al: Indoleamine 2,3-dioxygenase as a new target for malignant glioma therapy. Laboratory investigation. J Neurosurg 111: 230-237, 2009.

12. Muller AJ, DuHadaway JB, Donover PS, Sutanto-Ward E and Prendergast GC: Inhibition of indoleamine 2,3-dioxygenase, an immunoregulatory target of the cancer suppression gene Bin1, potentiates cancer chemotherapy. Nat Med 11: 312-319, 2005.

13. Zeng J, Cai S, Yi Y, et al: Prevention of spontaneous tumor development in a ret transgenic mouse model by ret peptide vaccination with indoleamine 2,3-dioxygenase inhibitor 1-methyl tryptophan. Cancer Res 69: 3963-3970, 2009.

14. Zhang KS, Li GC, He YW, et al: Curcumin inhibiting the expression of indoleamine 2,3-dioxygenase induced by IFN- $\gamma$ in cancer cells. Zhong Yao Cai 31: 1207-1211, 2008 (In Chinese).

15. Platanias LC: Mechanisms of type-I- and type-II-interferonmediated signalling. Nat Rev 5: 375-386, 2005.

16. Klampfer L, Huang J, Sasazuki T, Shirasawa S and Augenlicht L: Inhibition of interferon gamma signaling by the short chain fatty acid butyrate. Mol Cancer Res 1: 855-862, 2003.

17. Chon SY, Hassanain HH, Pine R and Gupta SL: Involvement of two regulatory elements in interferon-gamma-regulated expression of human indoleamine 2,3-dioxygenase gene. J Interferon Cytokine Res 15: 517-526, 1995.

18. Yamaguchi $\mathrm{H}$, Chen $\mathrm{CT}$, Chou $\mathrm{CK}$, et al: Adenovirus $5 \mathrm{E} 1 \mathrm{~A}$ enhances histone deacetylase inhibitors-induced apoptosis through Egr-1-mediated Bim upregulation. Oncogene 29: 5619-5629, 2010.

19. Sun PC, Tzao C, Chen BH, Liu CW, Yu CP and Jin JS: Suberoylanilide hydroxamic acid induces apoptosis and sub-G1 arrest of 320 HSR colon cancer cells. J Biomed Sci 17: 76, 2010.

20. Xu WS, Parmigiani RB and Marks PA: Histone deacetylase inhibitors: molecular mechanisms of action. Oncogene 26: 5541-5552, 2007.

21. Vigushin DM and Coombes RC: Histone deacetylase inhibitors in cancer treatment. Anticancer Drugs 13: 1-13, 2002.

22. Frew AJ, Johnstone RW and Bolden JE: Enhancing the apoptotic and therapeutic effects of HDAC inhibitors. Cancer Lett 280: 125-133, 2009.

23. Zong H, Yin B, Chen J, Ma B, Cai D and He X: Over-expression of c-FLIP confers the resistance to TRAIL-induced apoptosis on gallbladder carcinoma. Tohoku J Exp Med 217: 203-208, 2009.

24. Qing Y and Stark GR: Alternative activation of STAT1 and STAT3 in response to interferon-gamma. J Biol Chem 279: 41679-41685, 2004. 
25. Kobayashi N, Kubota K, Kato S, et al: FOXP3 ${ }^{+}$regulatory T cells and tumoral indoleamine 2,3-dioxygenase expression predicts the carcinogenesis of intraductal papillary mucinous neoplasms of the pancreas. Pancreatology 10: 631-640, 2010.

26. Munn DH: Indoleamine 2,3-dioxygenase, tumor induced tolerance and counter regulation. Curr Opin Immunol 18: 220-225, 2006

27. Urakawa H, Nishida Y, Nakashima H, Shimoyama Y, Nakamura $\mathrm{S}$ and Ishiguro $\mathrm{N}$ : Prognostic value of indoleamine 2,3-dioxygenase expression in high grade osteosarcoma. Clin Exp Metastasis 26: 1005-1012, 2009.

28. Dai W and Gupta SL: Regulation of indoleamine 2,3-dioxygenase gene expression in human fibroblasts by interferon-gamma. Upstream control region discriminates between interferon-gamma and interferon-alpha. J Biol Chem 265: 19871-19877, 1990.
29. Kadoya A, Tone S, Maeda H, Minatogawa Y and Kido R: Gene structure of human indoleamine 2,3-dioxygenase. Biochem Biophys Res Commun 189: 530-536, 1992.

30. Konan KV and Taylor MW: Importance of the two interferonstimulated response element (ISRE) sequences in the regulation of the human indoleamine 2,3-dioxygenase gene. J Biol Chem 271: 19140-19145, 1996.

31. Boehm U, Klamp T, Groot M and Howard JC: Cellular responses to interferon-gamma. Annu Rev Immunol 15: 749-795, 1997.

32. Jeong YI, Kim SW, Jung ID, et al: Curcumin suppresses the induction of indoleamine 2,3-dioxygenase by blocking the Janus-activated kinase-protein kinase Cdelta-STAT1 signaling pathway in interferon-gamma-stimulated murine dendritic cells. J Biol Chem 284: 3700-3708, 2009. 